\title{
Heparin nanomodification improves biocompatibility and biomechanical stability of decellularized vascular scaffolds
}

This article was published in the following Dove Press journal:

International Journal of Nanomedicine

23 November 2012

Number of times this article has been viewed

\author{
Yunming Tao ${ }^{1,2}$ \\ Tiehui $\mathrm{Hu}^{\prime}$ \\ Zhongshi Wu' \\ Hao Tang' \\ Yerong $\mathrm{Hu}^{\prime}$ \\ Qi Tan' \\ Chunlin $\mathrm{Wu}^{\prime}$ \\ 'Department of Thoracic and \\ Cardiovascular Surgery, Second \\ Xiangya Hospital of Central South \\ University, Changsha; ${ }^{2}$ Department of \\ Thoracic and Cardiovascular Surgery, \\ Ji'an Central People's Hospital, Ji'an, \\ Jiangxi Province, People's Republic of \\ China
}

Correspondence: Zhongshi Wu

Department of Thoracic and

Cardiovascular Surgery, Second Xiangya

Hospital of Central South University,

Changsha, Hunan Province 4100II,

People's Republic of China

Tel +8673185295108

Fax +8673185292133

Email taoyunming@।63.com

\begin{abstract}
Biocompatibility and biomechanical stability are two of the main obstacles limiting the effectiveness of vascular scaffolds. To improve the biomechanical stability and biocompatibility of these scaffolds, we created a heparin-nanomodified acellular bovine jugular vein scaffold by alternating linkage of heparin and dihydroxy-iron via self-assembly. Features of the scaffold were evaluated in vitro and in vivo. Heparin was firmly linked to and formed nanoscale coatings around the fibers of the scaffold, and the amount of heparin linked was about $808 \pm 86 \mu \mathrm{g} / \mathrm{cm}^{2}$ $\left(101 \pm 11 \mathrm{USP} / \mathrm{cm}^{2}\right)$ per assembly cycle. The scaffolds showed significantly strengthened biomechanical stability with sustained release of heparin for several weeks in vitro. Importantly, the modified scaffolds showed significantly reduced platelet adhesion, stimulated proliferation of endothelial cells in vitro, and reduced calcification in a subcutaneous implantation rat model in vivo. Heparin nanomodification improves the biocompatibility and biomechanical stability of vascular scaffolds.
\end{abstract}

Keywords: scaffolds, nanomodification, heparin, sustained release, biomechanical stability, biocompatibility

\section{Introduction}

Vascular grafts have been used widely in cardiovascular disease, cancer, trauma, organ transplantation, and microsurgical reconstruction. Vascular graft substitutes include autologous vessels, vascular allografts, vascular xenografts, and synthetic vascular grafts. ${ }^{1,2}$ Autologous vessels and vascular allografts are ideal alternative vessels, but an insufficient supply of donor vessels limits their widespread clinical application. ${ }^{3}$ Synthetic vascular grafts perform well in large vessel reconstruction, and there is a plentiful supply, but low graft patency greatly limits surgical utilization of small-caliber synthetic grafts because of thrombosis. ${ }^{1,4}$

We recently reported on in vivo use of decellularized bovine jugular vein (BJV) scaffolds to reconstruct pulmonary and right ventricular tissue in a dog model, with potential regeneration. ${ }^{5-7}$ The acellular vascular scaffolds were able to retain the tensile strength and unique extracellular matrix composition of native vessels, and thus could serve as an alternative blood vessel. ${ }^{8,9}$ Acellular BJV is a biological xenograft with a retained natural valve, and glutaraldehyde-treated BJV (Contegra ${ }^{\circledR}$, Medtronic Inc, Minneapolis, MN) provides a promising substitute for surgical valvular reconstruction of the right ventricular outflow tract. ${ }^{10-12}$ However, poor biocompatibility and biomechanical stability of acellular BJV scaffolds, including poor endothelialization, thrombus formation, aneurysm formation, and calcification, limit the clinical effectiveness of these scaffolds. ${ }^{10-12}$ 
Poor biocompatibility can induce thrombogenicity. Thrombus formation is one reason for transplant failure. ${ }^{5-7,13}$ To prevent early thrombosis after vascular xenograft implantation, systemic anticoagulant therapy is commonly used and this increases the risk of bleeding complications. ${ }^{13}$ A thromboresistant surface or endothelialization of a vascular graft can effectively prevent thrombosis. Establishing a thromboresistant surface is crucial to prevent formation of blood clots in the initial phase of graft implantation. ${ }^{14}$

Poor biomechanical stability can induce aneurysms. Formation of an aneurysm is another reason for transplant failure because of poor biomechanical stability of xenografts. ${ }^{10-12}$ Glutaraldehyde is used to improve the stability of a xenograft, but this procedure increases the cytotoxicity of xenografts and induces thrombogenicity. ${ }^{10-12}$

Heparin is a conventional anticoagulant and can be linked to the surface of a vascular graft. ${ }^{15}$ This approach can reduce thrombus formation without bleeding complications and also provide a substrate to bind growth factors, including basic fibroblast growth factor, epithelial growth factor, and vascular endothelial growth factor, which accelerate endothelialization and regeneration. ${ }^{14,16-18}$ Heparin can be linked to an extracellular matrix using a crosslinker such as glutaraldehyde, 1-(3-dimethylaminopropyl)-3ethylcarbodiimide hydrochloride (EDC), or chitosan, but the amount of heparin linked is small, and long-term results are not ideal. ${ }^{14,19-21}$ Much effort has been devoted to improving the antithrombogenicity of xenogeneic skin grafts by surface modification using anticoagulant molecules. Dai et al reported that complex multilayers of iron polysaccharide, which are used to modify a nitinol surface, provide a stable thromboresistant surface with good biocompatibility. ${ }^{22}$

In the present study, our main objective was to develop a decellularized vascular scaffold with heparin nanomodification, and to improve the biocompatibility and biomechanical stability of acellular vascular scaffolds. Stable heparin-nanomodified scaffolds were created by alternating linkage of heparin and dihydroxy-iron (DHI) to the scaffolds via layer-by-layer self-assembly. Furthermore, we evaluated the biocompatibility and biomechanical stability of the modified vascular scaffold in vitro and in vivo, using an unmodified counterpart as a control. Heparin-nanomodified scaffolds could provide a stable antithrombogenic surface and significantly improve biomechanical stability and biocompatibility compared with unmodified scaffolds. The results of this study suggest a new method for developing tissue-engineered vascular grafts.

\section{Materials and methods Materials and reagents}

Heparin sodium (molecular weight 10,000-15,000, $125 \mathrm{USP} / \mathrm{mg}$ ) was purchased from Sigma Ltd (St Louis, MO) and ferric chloride hexahydrate $\left(\mathrm{FeCl}_{3} \cdot 6 \mathrm{H}_{2} \mathrm{O}\right.$, molecular weight 270.8) was obtained from Tianda Chemical (Tianjing, China).

Preparation of DHI $\left(\left[\mathrm{Fe}(\mathrm{OH})_{2}\right]^{+}\right)$solution was as follows. $\mathrm{FeCl}_{3} \cdot 6 \mathrm{H}_{2} \mathrm{O}$ was dissolved in deionized water, and its $\mathrm{pH}$ was slowly adjusted to 3.0 with $\mathrm{NaOH}$ solution while stirring (molar ratio of $\mathrm{NaOH}$ to $\mathrm{FeCl}_{3}$ is 2 to 1 ). Its concentration was then adjusted to $0.05 \mathrm{~mol} / \mathrm{L}$ with deionized water.

Preparation of acellular BJV scaffolds was as follows. BJVs from 300-500 kg cattle beasts were obtained from a local slaughterhouse and stored in chilled normal saline. The loose connective tissue and fat on the outer surface of the BJVs was removed in the laboratory. The multistep detergent-enzymatic decellularization procedure has been described in detail previously. ${ }^{5-7}$

\section{Modification procedure}

Acellular BJV scaffolds were modified as follows. First, the acellular BJV scaffolds were trimmed to the desired shape and size, immersed in $0.05 \mathrm{~mol} / \mathrm{L}$ DHI solution for $10 \mathrm{~min}-$ utes at room temperature, and then rinsed three times with $0.9 \% \mathrm{NaCl}$ solution for 5 minutes. Next, the scaffolds were immersed in $5 \mathrm{mg} / \mathrm{mL}$ heparin solution containing $0.9 \% \mathrm{NaCl}$ for 5 minutes at room temperature, followed by washing three times with $0.9 \% \mathrm{NaCl}$ solution for 5 minutes. Subsequently, the scaffolds were immersed in $0.05 \mathrm{~mol} / \mathrm{L}$ DHI solution for 5 minutes at room temperature. Finally, after completion of the desired number of cycles, the scaffolds were removed, rinsed with $0.9 \% \mathrm{NaCl}$ solution, and stored in $5 \mathrm{mg} / \mathrm{mL}$ heparin solution containing $0.9 \% \mathrm{NaCl}$ at $4{ }^{\circ} \mathrm{C}$ for further use.

\section{Quantification of linked heparin}

The toluidine blue colorimetric method was used to measure the initial and residual concentration of heparin solution before and after each assembly cycle, and the amount of linked heparin was calculated. ${ }^{23,24}$ In brief, acellular BJV scaffolds were cut into square sheets $(1 \mathrm{~cm} \times 1 \mathrm{~cm}, \mathrm{n}=12)$ and modified as described above. The initial concentration of heparin solution was detected by the toluidine blue colorimetric method first, then the residual concentration of heparin solution was detected after the sheets were removed from the heparin solution during each assembly cycle. Absorbance was recorded at $630 \mathrm{~nm}$ using an ultraviolet spectrophotometer (UV-2450 Shimadzu Corporation, Tokyo, Japan), and the 
amount of linked heparin per $\mathrm{cm}^{2}$ per cycle was calculated. The amount of linked heparin was calculated as (the initial concentration of heparin solution - the residual concentration of heparin solution) $\times$ the volume of heparin solution.

\section{Surface ultrastructure}

Scanning electron microscopy (SEM, Nova NanoSEM 230, FEI Company, Hillsboro, OR) was used to evaluate the variances in surface ultrastructure between unmodified and heparin/DHI-modified BJV scaffolds. In brief, acellular BJV scaffolds were cut into vessel sheets $(1 \mathrm{~cm} \times 1 \mathrm{~cm})$ and modified by one, four, seven, ten, and 13 cycles of heparin/ DHI coating as described earlier, with unmodified BJV scaffolds were used as the control. All samples were fixed in 4\% glutaraldehyde for 24 hours, rinsed in phosphate-buffered saline, postfixed in $1 \% \mathrm{OsO}_{4}$ for 2 hours, followed by a brief wash with distilled water and then dehydration with graded ethanol. The samples were then critical-point dried, mounted luminal side up, sputter-coated with gold, and examined using SEM (JEOL-6490 LV, JEOL, Tokyo, Japan).

\section{Histological characteristics}

Linked heparin in tissue sections can be stained with toluidine blue and visualized using light microscopy. ${ }^{14,25}$ Briefly, the formaldehyde-fixed BJV scaffold samples modified or not modified by seven cycles heparin/DHI were embedded in paraffin and cut into slices. The histological sections were deparaffinized and hydrated routinely, then stained with $0.1 \%$ toluidine blue for 10 minutes. Next, they were dehydrated and mounted in a routine manner. Photographs were taken under a light microscope (Nikon E600, Tokyo, Japan).

\section{Washing test}

To study the binding force of the heparin/DHI coating on the surface of the BJVs, the shaken-wash model was used, which mimics resting heart rate, body temperature, and exposure of the heparin/DHI coatings to fluid flow stress. ${ }^{22}$ In brief, acellular BJV scaffolds were cut into square sheets ( $1 \mathrm{~cm} \times 1 \mathrm{~cm}, 10$ samples each) and modified using four, seven, and ten cycles of heparin/DHI as described above. Each sheet was subjected to a tangential shaking wash in $40 \mathrm{~mL}$ of phosphate-buffered saline at $37^{\circ} \mathrm{C}$, and $0.2 \mathrm{~mL}$ samples of the washed solution were removed after washing for one day and one, 2, 4, 6, and 8 weeks. After removal, the heparin concentration in the samples was determined using the toluidine blue colorimetric method. Absorbance was recorded as described above, and the cumulative amount of heparin released was calculated. The ultrastructure of the luminal surface of the BJV scaffold modified by seven cycles of heparin/DHI was examined after 8 weeks of washing as described above.

\section{Biomechanical stability}

A tensile test was used to evaluate the biomechanical stability of the heparin/DHI-modified scaffolds. ${ }^{6,7}$ Briefly, 20 pieces of acellular BJV scaffolds which were more than $1 \mathrm{~cm}$ away from the vein valve sinus were cut transversely into six portions. Five portions of scaffolds were transversely cut into vessel sheets $(1 \mathrm{~cm} \times 4 \mathrm{~cm})$ and modified by one, four, seven, ten, and 13 cycles of heparin/DHI as described above, and the other scaffolds were used as controls. The elastic modulus, maximum load, and maximum tensile stress of the modified and unmodified scaffolds were tested using a tensile test machine (Instron Co, Norwood, MA).

\section{Antithrombogenic activity in vitro}

The activated partial thrombin time (APTT) and prothrombin time are used widely to evaluate the antithrombogenic activity of biomaterials. ${ }^{22}$ In brief, BJV scaffolds modified by seven cycles of heparin/DHI were cut into square sheets $(1 \mathrm{~cm} \times 1 \mathrm{~cm})$, and every sheet was washed in $40 \mathrm{~mL}$ of phosphate-buffered saline at $37^{\circ} \mathrm{C}$ under $80 \mathrm{rpm}$ shaking conditions. The sheets were removed after one day and one, 4, 6, and 8 weeks of washing (eight samples each), and then rinsed three times with phosphate-buffered saline for 3 minutes. Unmodified BJV scaffolds were also cut into sheets $(n=8)$ and used as controls. Each sheet was then placed carefully in a tube containing $1 \mathrm{~mL}$ of citrateanticoagulated blood (obtained from volunteer donors) at $37^{\circ} \mathrm{C}$ for 60 minutes, and the blood was next centrifuged for 15 minutes at $3000 \mathrm{rpm}$ to obtain the plasma. The APTT and prothrombin time of the resulting plasma were detected using an automatic blood coagulation analyzer (CA6000, Sysmex Ltd, Kobe, Japan).

\section{Platelet adhesion in vitro}

The biocompatibility of biomaterials can be investigated by evaluation of platelet adhesion. ${ }^{22,26}$ Briefly, platelet-rich plasma was generated by centrifugation for 15 minutes at $750 \mathrm{rpm}$. Scaffolds modified by seven cycles of heparin/ DHI were then cut into square sheets $(1 \mathrm{~cm} \times 1 \mathrm{~cm}, \mathrm{n}=6)$, and every sheet was positioned in contact with $1 \mathrm{~mL}$ of platelet-rich plasma for 3 hours in a humidified $5 \% \mathrm{CO}_{2}$ air atmosphere at $37^{\circ} \mathrm{C}$. Unmodified scaffold sheets $(n=6)$ were used as the control. The specimens were fixed in $4 \%$ glutaraldehyde solution for 24 hours, then washed in 
phosphate-buffered saline three times and dehydrated using graded ethanol. Next, the specimens were dried in a vacuum dryer, sputter-coated with gold, and observed using SEM.

\section{Cytotoxicity assay in vitro}

An in vitro cytotoxicity assay was used to evaluate the cytotoxicity of the BJV scaffolds modified with heparin/DHI. In brief, BJV scaffolds modified with seven cycles of heparin/ DHI were sheared into $1.0 \mathrm{~cm}$ diameter disks $(\mathrm{n}=48)$ and sterilized using gamma-ray irradiation at $20 \mathrm{KGy}$. Human EA.hy926 endothelial cells $\left(1 \times 10^{5}\right.$ cells $)$ from a spontaneously transformed human umbilical vein endothelial cell line (ScienCell Co, San Diego, CA) were seeded onto the luminal surface of the modified BJV scaffolds in 48-well culture plates. ${ }^{18,27}$ The disks were incubated in a humidified atmosphere of $5 \% \mathrm{CO}_{2}$ and $95 \%$ air at $37^{\circ} \mathrm{C}$ for one, 4,7 , and 10 days ( 12 disks each). Unmodified BJVs $(n=48)$ were used as the control. Dulbecco's Modified Eagle's Medium (Hyclone Ltd, Logan, UT) supplemented with 10\% fetal bovine serum (Hyclone Ltd) was used as the culture medium and changed routinely every 2 days.

At the end of the culture period, each group of disks was divided into two portions. One portion was subjected to MTT assay as follows. The disks were incubated for 4 hours in serum-free medium with $0.8 \mathrm{mg} / \mathrm{mL}$ MTT (Sigma Ltd), then washed briefly with phosphate-buffered saline, followed by shaking in $1 \mathrm{~mL}$ of dimethyl sulfoxide (Sigma Ltd) for 10 minutes. Next, $0.2 \mathrm{~mL}$ samples of the resulting solution were transferred into 96-well plates, and absorbance was recorded at $560 \mathrm{~nm}$ using a microplate spectrophotometer (Bio-Tek Instruments Inc, Winooski, VT). The other portion of the disks was assayed by light microscopy. For this examination, the disks were stained with $\mathrm{DioC}_{18}$ solution (Beyotime Institute of Biotechnology, Haimen, China) for 10 minutes, and photographs were taken under a fluorescence microscope (E600, Nikon Corporation, Tokyo, Japan). ${ }^{28}$

\section{Anticalcification in a rat model}

Subcutaneous implantation was used to evaluate calcification in the BJV scaffolds modified using heparin/DHI. Briefly, 20 Sprague-Dawley rats (140-150 g) were obtained from the animal department of Central South University. Sheets of BJV scaffolds $(1 \mathrm{~cm} \times 1 \mathrm{~cm}, \mathrm{n}=20)$ modified using seven cycles of heparin/DHI or not (control scaffold sheets) were subcutaneously implanted symmetrically on both sides of the rat spine and retrieved after 30 days $(n=10)$ or 60 days $(n=10)$. Rat handling complied with the requirements for care and use of animals according to National Institutes of
Health and Chinese guidelines. The samples retrieved were dried to a constant weight in a desiccator oven and hydrolyzed with $\mathrm{HNO}_{3}(10 \mathrm{~mL}, 0.75 \mathrm{~mol} / \mathrm{L})$ at $70^{\circ} \mathrm{C}$ for 15 hours. After centrifugation ( 15 minutes, $3000 \mathrm{rpm}$ ), the fluid was removed and diluted, and the calcium content was determined using a fast sequential atomic absorption spectrometer (Varian AA240FS, Varian Inc, Palo Alto, CA) ${ }^{29}$

\section{Statistical analysis}

All values are expressed as the mean \pm standard deviation. The statistical analysis was performed using analysis of variance followed by the Student-Newman-Keuls test to compare the groups in a pairwise manner. The statistical analysis was performed using the Statistical Package for Social Sciences, Windows version 18.0 (SPSS Inc, Chicago, IL). A $P<0.05$ was considered to be statistically significant.

\section{Results}

\section{Gross appearance}

The gross appearance of the scaffolds is shown in Figures 1 and 2. The vessel walls and valves with unmodified BJV scaffolds (Figure 1A) and the scaffolds modified by heparin/ DHI (Figure 1B) had a similar shape, flexibility, and texture. However, the color of the luminal and outer surfaces of the BJV scaffolds modified by heparin/DHI changed from white to golden yellow, whereas the middle layer of the modified scaffolds remained white (Figure 2). The intensity of the golden yellow color appeared to increase with an increase in the number of assembly cycles (Figure 2).

\section{Quantification of linked heparin}

We detected the initial and residual concentrations of heparin solution using the toluidine blue colorimetric method
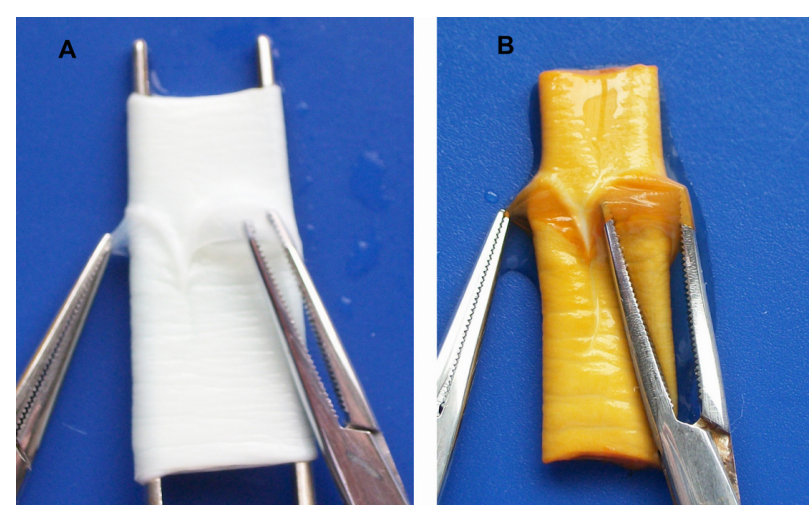

Figure I Gross appearance of scaffolds. (A) Gross appearance of unmodified decellularized BJV scaffolds. (B) Gross appearance of heparin/DHI-modified decellularized BJV scaffolds.

Abbreviations: BJV, bovine jugular vein; DHI, dihydroxy-iron. 


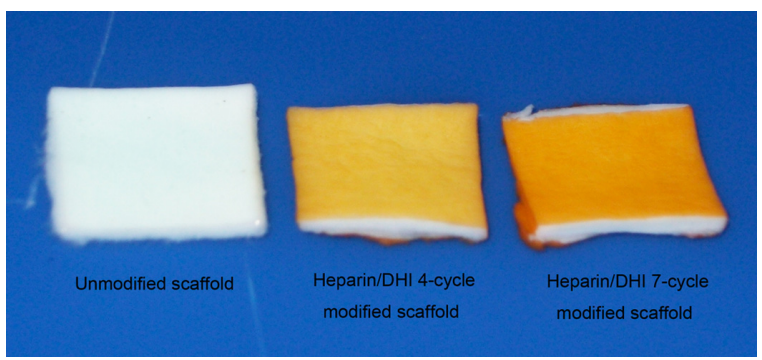

Figure 2 Gross appearance of decellularized BJV scaffold sheets modified or not modified by four and seven cycles of heparin/DHI, respectively.

Abbreviations: BJV, bovine jugular vein; DHI, dihydroxy-iron.

and calculated the amount of linked heparin. The results show that the average amount of linked heparin was about $808 \pm 86 \mu \mathrm{g} / \mathrm{cm}^{2}\left(101 \pm 11 \mathrm{USP} / \mathrm{cm}^{2}\right)$ per cycle, and the mean amount of linked heparin was about $3.23 \pm 0.34,5.46 \pm 0.60$, and $8.80 \pm 0.86 \mathrm{mg} / \mathrm{cm}^{2}$ after four, seven, and ten cycles, respectively.

\section{Surface ultrastructure and histological characteristics}

SEM images of the ultrastructure of the luminal surface (Figure 3) showed that heparin and DHI were linked to and formed nanoscale coatings around the fibrils of the scaffolds. The coatings of the BJV scaffolds modified by four, seven, and ten cycles of heparin/DHI were uniform, but the coatings of scaffolds modified by 13 cycles of heparin/ DHI were not uniform. We compared the diameters of fibrils from the BJV scaffolds modified by heparin/DHI with those of their unmodified counterparts, and the results show that the thickness of the heparin/DHI coatings increased with an increase in the number of assembly cycles; the thickness of one-cycle heparin/DHI coating was about $25 \mathrm{~nm}$, and the thicknesses of BJV scaffolds modified by four, seven, and ten cycles of heparin/DHI coating were about 95, 160, and $260 \mathrm{~nm}$, respectively. However, if assembly involved more than seven cycles, adjacent fibrils could be fused with each other by the heparin/DHI complexes.

Photographs of sections stained using toluidine blue (Figure 4) showed that both the outer and luminal layers of the heparin/DHI-modified scaffolds (Figure 4A) were stained deeply, whereas the middle layers of BJV scaffolds modified by heparin/DHI were stained lightly but more deeply than in their unmodified counterparts (Figure 4B).

\section{Stability of heparin/DHI coatings}

The modified scaffolds still retained their golden yellow color after 8 weeks of washing. The ultrastructure of the luminal surface of the scaffolds modified by heparin/DHI after 8 weeks of washing is shown in Figure 5. There was a large amount of heparin left in the scaffold, and the remains of the heparin/ DHI coating could be found around the fibrils. Cumulative release of heparin is shown in Table 1 . The results indicate that the heparin/DHI coating released heparin in a sustained manner for at least 8 weeks. There was no significant difference in the amount of heparin released between the scaffolds modified by heparin/DHI using different cycles.

\section{Strengthened biomechanical stability}

The results of the tensile test are shown in Table 2 and Figure 6. Compared with the unmodified scaffolds, the elastic
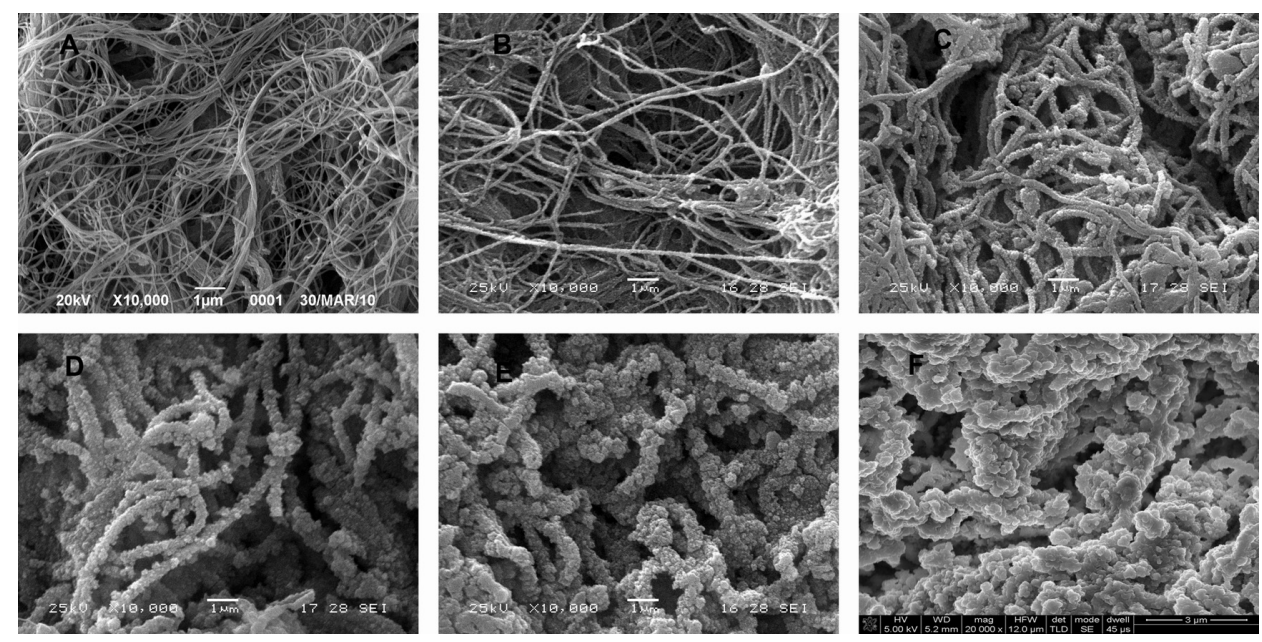

Figure 3 Luminal surface ultrastructure of scaffolds. (A) SEM image of unmodified scaffold $(10,000 \times)$. (B) SEM image of a scaffold modified by one cycle of heparin/DHI $(10,000 \times)$. (C) SEM image of scaffold modified by four cycles of heparin/DHI (I0,000X). (D) SEM image of scaffold modified by seven cycles of heparin/DHI (I0,000 $)$. (E) SEM image of scaffold modified by ten cycles of heparin/DHI (I0,000X). (F) SEM image of scaffold modified by one cycle of heparin/DHI (20,000X).

Abbreviations: DHI, dihydroxy-iron; SEM, scanning electron microscope. 


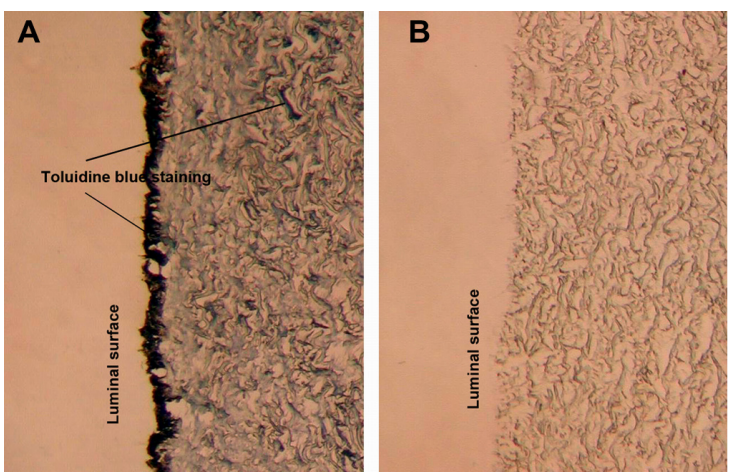

Figure 4 Toluidine blue staining of scaffolds $(200 \times)$. (A) Toluidine blue staining shows the outer and middle layers of BJV scaffolds modified by heparin/DHI stained by toluidine blue, with the outer layer stained more deeply than the middle layer. The arrows indicate positive staining. (B) Toluidine blue staining shows that the unmodified BJV scaffolds could not be stained by toluidine blue.

Abbreviations: BJV, bovine jugular vein; DHI, dihydroxy-iron.

modulus, maximum load, and maximum tensile stress of the heparin/DHI-modified scaffolds were significantly increased. There were significant differences between the unmodified and heparin/DHI-modified scaffold groups $(P<0.01)$. The biomechanical stability of the modified scaffolds increased with an increase in the number of assembly cycles. However, biomechanical stability was not significantly increased after ten cycles.

\section{Increased antithrombogenic activity}

The results of the coagulation activity assay are shown in Table 3. The prothrombin time and APTT of the unmodified scaffolds were within the normal reference range, but above

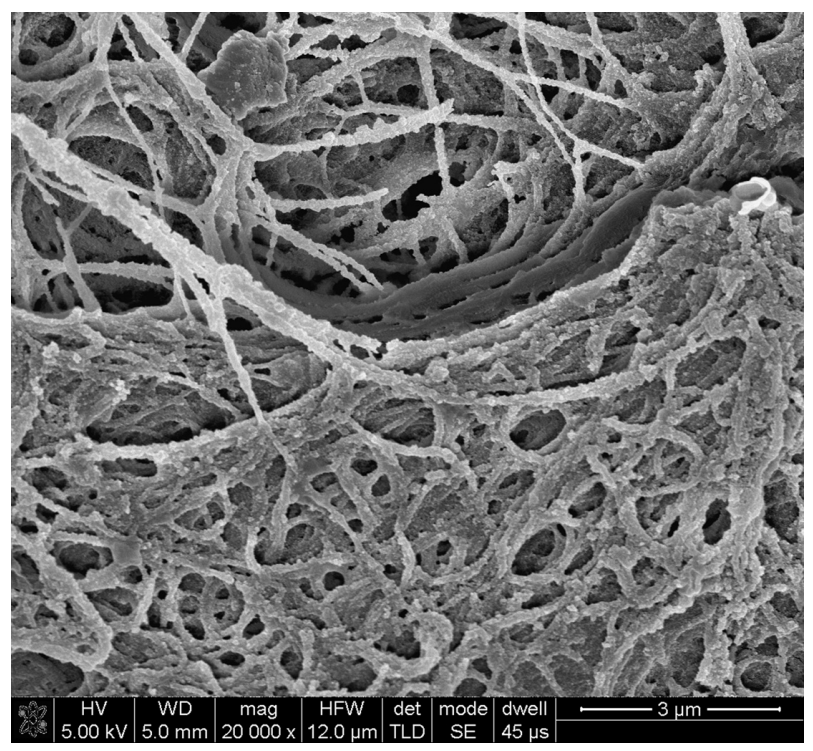

Figure 5 Ultrastructure of the luminal surface of heparin/DHI-modified BJV scaffolds after 8 weeks of washing.

Abbreviations: BJV, bovine jugular vein; DHI, dihydroxy-iron. the upper limit of the normal range in all the heparin/DHImodified scaffold groups. It is important to note that the prothrombin time and APTT of the modified sheets removed after one day and one week of washing were above the upper detection limit of the blood coagulation analyzer $(>120$ seconds for prothrombin time and $>180$ seconds for APTT). There was a significant difference in antithrombogenic activity between the unmodified and heparin/DHI-modified scaffold groups, regardless of washing duration $(P<0.01)$.

\section{Reduced platelet adhesion}

SEM images of platelets adhered to the luminal surface (Figure 7) indicate that the heparin/DHI-modified scaffolds drastically decreased surface platelet adhesion compared with their unmodified counterparts. Large numbers of platelets adhered to and aggregated in some regions of the unmodified sheets (Figure 7B), but few platelets were found on the luminal surfaces of the heparin/DHI-modified sheets (Figure 7A). Mean platelet counts per $10,000 \mu \mathrm{m}^{2}$ area for the heparin/DHI-modified and unmodified scaffolds were $8 \pm 4$ and $48 \pm 16$, respectively. The difference between the two groups was significant $(P<0.01)$.

\section{Stimulated proliferation of endothelial cells}

Photographs of $\mathrm{DioC}_{18}$ staining are shown in Figure 6. The results of this assay show that human EA.hy926 endothelial cells could adhere to and proliferate on the luminal surface of both heparin/DHI-modified and unmodified scaffolds. The number of endothelial cells on the luminal surface of a heparin/DHI-modified sheet (Figure 8E) was less than the number on the luminal surface of an unmodified sheet (Figure 8A) after one day of incubation. After 4 days of incubation, the number of endothelial cells on the luminal surface of the modified sheet (Figure 8F) was very similar to that on the surface of its unmodified counterpart (Figure 8B). However, the number of endothelial cells on the luminal surface of the unmodified scaffold (Figure 8C and D) was less than the number on the luminal surface of the heparin/ DHI-modified scaffold sheet (Figure $8 \mathrm{G}$ and $\mathrm{H}$ ) after incubation for 7 and 10 days. In particular, at 10 days of incubation, the morphology of the endothelial cells on the luminal surface of the unmodified scaffold sheets changed to being smaller and thinner than that on the heparin/DHI-modified scaffold sheets.

The MTT assay showed similar results. Mean OD values were $0.21 \pm 0.04,0.39 \pm 0.03,0.58 \pm 0.0,6$ and $0.65 \pm 0.08$ for heparin/DHI-modified sheets after incubation 
Table I Cumulative release of heparin from heparin/DHI modified scaffolds $(n=10)$

\begin{tabular}{lllllll}
\hline Group & \multicolumn{4}{l}{ Cumulative release of heparin $\left(\mu \mathrm{g} / \mathbf{c m}^{2}\right)$} & & \\
\cline { 2 - 7 } & I day & I week & 2 weeks & 4 weeks & 6 weeks & 8 weeks \\
\hline 4-cycle modified scaffold & $257 \pm 38$ & $368 \pm 53$ & $689 \pm 79$ & $1298 \pm 161$ & $1678 \pm 179$ & $1877 \pm 214$ \\
7-cycle modified scaffold & $28 I \pm 43$ & $379 \pm 60$ & $721 \pm 81$ & $1317 \pm 157$ & $1685 \pm 187$ & $1915 \pm 228$ \\
I0-cycle modified scaffold & $274 \pm 45$ & $358 \pm 67$ & $712 \pm 87$ & $1309 \pm 175$ & $1701 \pm 233$ & $2011 \pm 273$ \\
\hline
\end{tabular}

Note: $P>0.05$, there was no significant difference among all heparin/DHI-modified scaffold groups.

Abbreviation: $\mathrm{DH}$, dihydroxy-iron.

for one, 4, 7, and 10 days, respectively. Corresponding mean OD values for the unmodified sheets were $0.32 \pm 0.03$, $0.40 \pm 0.04,0.38 \pm 0.05$, and $0.28 \pm 0.08$ after incubation for one, 4,7 , and 10 days, respectively. There were significant differences between OD values obtained for unmodified and heparin/DHI-modified scaffolds after incubation for one, 7 , and 10 days $(P<0.01)$.

\section{Reduced calcification}

The modified scaffolds retrieved after 30 or 60 days retained their golden yellow color (Figure 9). Mean calcium content (Figure 10) after 30 days of incubation for the heparin/DHImodified and unmodified BJV scaffolds was $8.5 \pm 1.9$ and $46.6 \pm 3.7 \mu \mathrm{g} / \mathrm{mg}$ dry weight, respectively, and at 60 days was $12.5 \pm 6.8$ and $112.6 \pm 16.9 \mu \mathrm{g} / \mathrm{mg}$ dry weight. There was a significant difference in calcium content between the heparin/DHI-modified and unmodified scaffolds at both time points $(P<0.01)$.

\section{Discussion}

In the current study, a stable heparin/DHI-nanomodified acellular vascular xenograft scaffold was developed by alternating dative covalent linkage of DHI ions and heparin molecules onto BJV scaffolds using layer-by-layer self-assembly. The mechanisms for establishing heparin/DHI complexes may be as follows. Iron ion, an essential trace element in the human body, is a typical transition metal cation that accepts electron pairs. DHI ions are formed when $\mathrm{FeCl}_{3}$ solution mixes slowly with $\mathrm{NaOH}$ solution, and the chemical equation is
$\mathrm{Fe}^{3+}+2 \mathrm{OH}^{-}=\left[\mathrm{Fe}(\mathrm{OH})_{2}\right]^{+}$. Heparin, a highly sulfated linear polysaccharide, contains large numbers of hydroxyl, amino, carboxyl, sulfonyl, and other groups, and these groups are typical Lewis bases that donate electron pairs. ${ }^{15,30,31}$ Positively charged DHI ions can be combined with negatively charged sulfonic or carboxylic acids of heparin molecules by ionic bonds, and they can also be chemically bonded with the carboxyl, sulfonyl, hydroxyl, or amino groups of heparin molecules by dative covalent bonds. Hence, heparin/DHI complexes are formed when heparin molecules and DHI ions chemically bond with each other. The extracellular matrix, which consists of collagen fibers, elastic fibers, fibronectin, laminin, glycosaminoglycan, and proteoglycan, also contains large numbers of carboxyl, hydroxyl, amino, and other groups that can donate electron pairs. ${ }^{15,32}$ As described above, DHI ions can also be chemically bonded to the extracellular matrix by dative covalent bonds.

The self-assembly procedure may occur as follows. Firstly, when acellular BJV scaffolds are immersed in DHI solution, DHI ions are chemically combined with the carboxyl, hydroxyl, or amino groups of the extracellular matrix by dative covalent bonds and linked to the scaffolds. Secondly, when the scaffolds are immersed in heparin solution, the carboxyl, sulfonyl, hydroxyl, or amino groups on the heparin molecules are chemically combined with the DHI ions linked into the extracellular matrix by dative covalent bonds, and heparin molecules are linked onto the scaffolds. Thirdly, when the scaffolds are immersed in DHI solution again, DHI ions are chemically combined with the remaining carboxyl, sulfonyl,

Table 2 Biomechanical stability of unmodified and heparin/DHI-modified scaffolds $(n=20)$

\begin{tabular}{llll}
\hline Group & Elastic modulus $\mathbf{( M P a )}$ & Maximum load $(\mathbf{N})$ & Maximum tensile press $(\mathbf{M P a})$ \\
\hline Unmodified scaffold* & $5.01 \pm 0.23$ & $29.78 \pm 1.81$ & $3.16 \pm 0.18$ \\
I-cycle modified scaffold & $5.57 \pm 0.21$ & $32.27 \pm 1.21$ & $3.72 \pm 0.16$ \\
4-cycle modified scaffold & $5.92 \pm 0.24$ & $34.29 \pm 1.08$ & $4.17 \pm 0.17$ \\
7-cycle modified scaffold & $6.23 \pm 0.21$ & $35.43 \pm 1.15$ & $4.46 \pm 0.15$ \\
I0-cycle modified scaffold** & $6.48 \pm 0.19$ & $36.75 \pm 0.97$ & $4.71 \pm 0.14$ \\
I3-cycle modified scaffold** & $6.61 \pm 0.16$ & $36.94 \pm 0.86$ & $4.85 \pm 0.12$ \\
\hline
\end{tabular}

Notes: $* P<0.01$ versus heparin/DHI-modified scaffolds, there was a significant difference between unmodified and heparin/DHI-modified scaffolds; $* * P>0.05$, there was no significant difference between the I0-cycle and I3-cycle-modified scaffold groups.

Abbreviation: $\mathrm{DHI}$, dihydroxy-iron. 

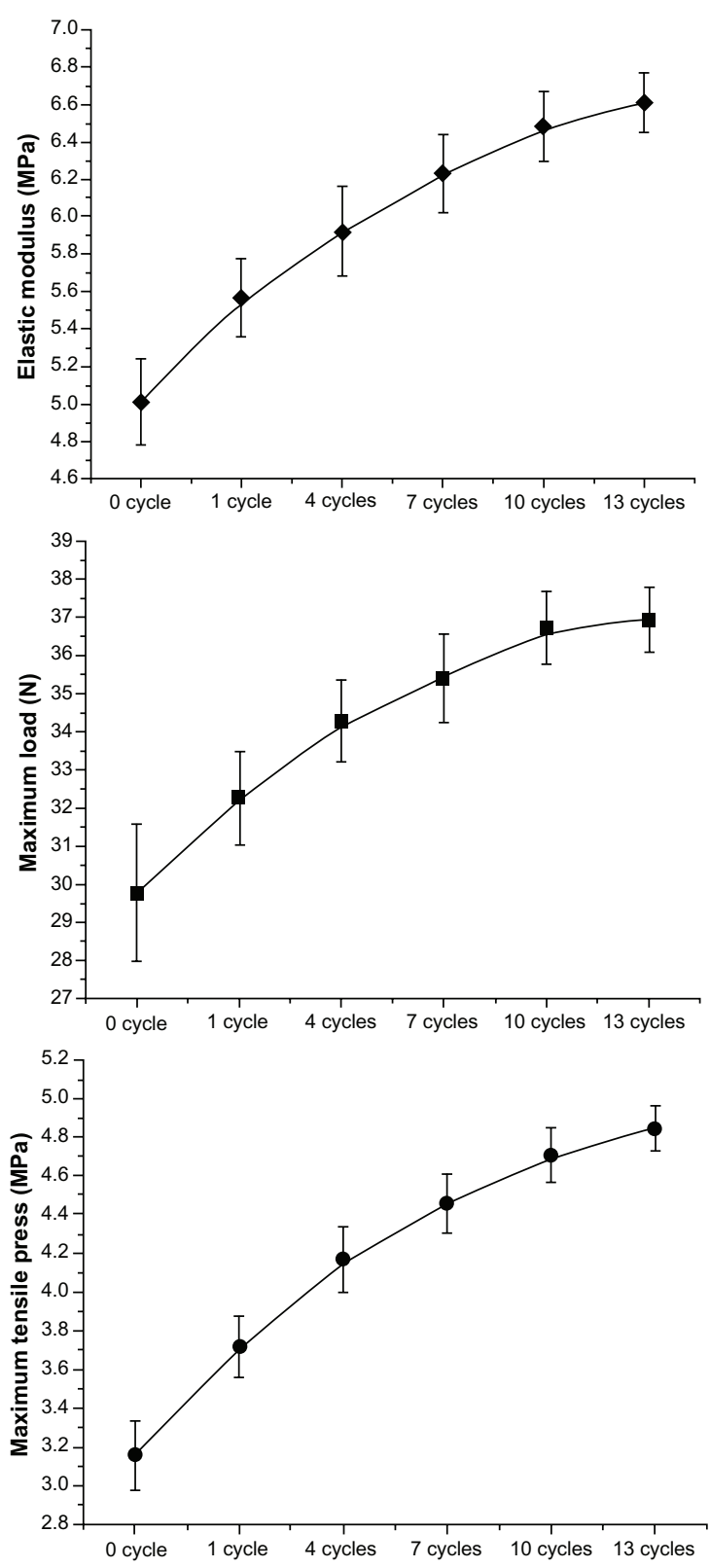

Figure 6 Line graphs showing tensile test results.

Note: Tensile curves show that the elastic modulus, maximum load, and maximum tensile stress of heparin/DHI-modified scaffolds increased along with an increase in the number of assembly cycles, but with no significant increase after ten cycles of nanomodification.

Abbreviation: $\mathrm{DHI}$, dihydroxy-iron.

hydroxyl, or amino groups of the linked heparin molecules in the extracellular matrix and linked to the scaffolds. Finally, after completion of several cycles, large numbers of heparin molecules and DHI ions are alternately linked to scaffolds and form heparin/DHI complex coatings around the fibrils.

In this study, quantification showed that the average amount of linked heparin was about $808 \pm 86 \mu \mathrm{g} / \mathrm{cm}^{2}(101 \pm 11$ $\mathrm{USP} / \mathrm{cm}^{2}$ ) per assembly cycle, and the total amount of linked heparin was about $8.80 \pm 0.86 \mathrm{mg} / \mathrm{cm}^{2}\left(1040 \pm 110 \mathrm{USP} / \mathrm{cm}^{2}\right)$
Table 3 Prothrombin time and APTT of unmodified and heparin/ DHI-modified scaffolds $(n=8)$

\begin{tabular}{lll}
\hline Group & PT/s & APTT/s \\
\hline Unmodified scaffold* & $11.8 \pm 1.5$ & $21.2 \pm 3.4$ \\
Modified scaffold $(\mathrm{I} \mathrm{d})^{\mathrm{a}}$ & $>120$ & $>180$ \\
Modified scaffold $(\mathrm{I} \mathrm{w})^{\mathrm{b}}$ & $>120$ & $>180$ \\
Modified scaffold $(4 \mathrm{w})^{\mathrm{c}}$ & $82.3 \pm 7.2$ & $112.5 \pm 13.1$ \\
${\text { Modified scaffold }(6 \mathrm{w})^{\mathrm{d}}}_{\text {Modified scaffold }(8 \mathrm{w})^{\mathrm{e}}}$ & $46.7 \pm 3.6$ & $84.7 \pm 8.9$ \\
\hline
\end{tabular}

Notes: anodified scaffold (I d), heparin/DHI-modified scaffolds that were removed after one day of washing; bmodified scaffold (I w), heparin/DHI-modified scaffolds that were removed after one week of washing; ' $m$ odified scaffold ( 4 w), heparin/DHImodified scaffolds that were removed after 4 weeks of washing; ${ }^{d}$ modified scaffold $(6 \mathrm{w})$, heparin/DHI-modified scaffolds that were removed after 6 weeks of washing; ${ }^{e}$ modified scaffold $(8 \mathrm{w})$, heparin/DHI-modified scaffolds that were removed after 8 weeks of washing; $* P<0.01$ versus heparin/DHI-modified scaffolds, indicates a significant difference between unmodified and modified scaffolds.

Abbreviations: APTT, activated partial thrombin time; PT, prothrombin time; DHI, dihydroxy-iron.

after ten cycles. In contrast, only $6.21 \pm 2.03 \mathrm{USP} / \mathrm{cm}^{2}$ of heparin was linked to the extracellular matrix when EDC was used as the crosslinker. ${ }^{14}$ These results indicate that more heparin molecules are linked to the extracellular matrix when using DHI than when using EDC. Our recent test results show that the average amount of linked iron ion was about $178 \pm 46 \mu \mathrm{g} / \mathrm{cm}^{2}$ per assembly cycle. ${ }^{33}$ Iron ion is an essential trace element used by the human body to produce red blood cells, and DHI is nontoxic to humans.

Heparin/DHI coatings could be visualized directly using SEM, which suggests that a large number of heparin molecules were linked to the scaffolds. SEM images of the luminal surface ultrastructure show that the thickness of a one-cycle heparin/DHI coating was about $25 \mathrm{~nm}$, suggesting that heparin/DHI complex coatings are nanoscale films. ${ }^{34,35}$ Increasing the number of assembly cycles should increase the thickness of the heparin/DHI coating, and if the number of assembly cycles increased enough, the heparin/DHI complexes would fill up the spaces between adjacent fibrils and form a microscale coating on the surface of the scaffold.

The current results show that both the outer and luminal layers of the heparin/DHI-modified vascular scaffold were stained deeply by toluidine blue, whereas the middle layer of the modified scaffold was stained lightly but more deeply than the unmodified scaffolds. These observations suggest that the heparin molecules were mainly linked to the surface of the scaffolds, with a little heparin linked to the middle layer.

The results of the tensile test show that the elastic modulus, maximum load, and maximum tensile stress in the heparin/DHI-modified scaffolds were increased significantly compared with unmodified scaffolds. Our findings suggest that nanomodification with heparin/DHI increased the 

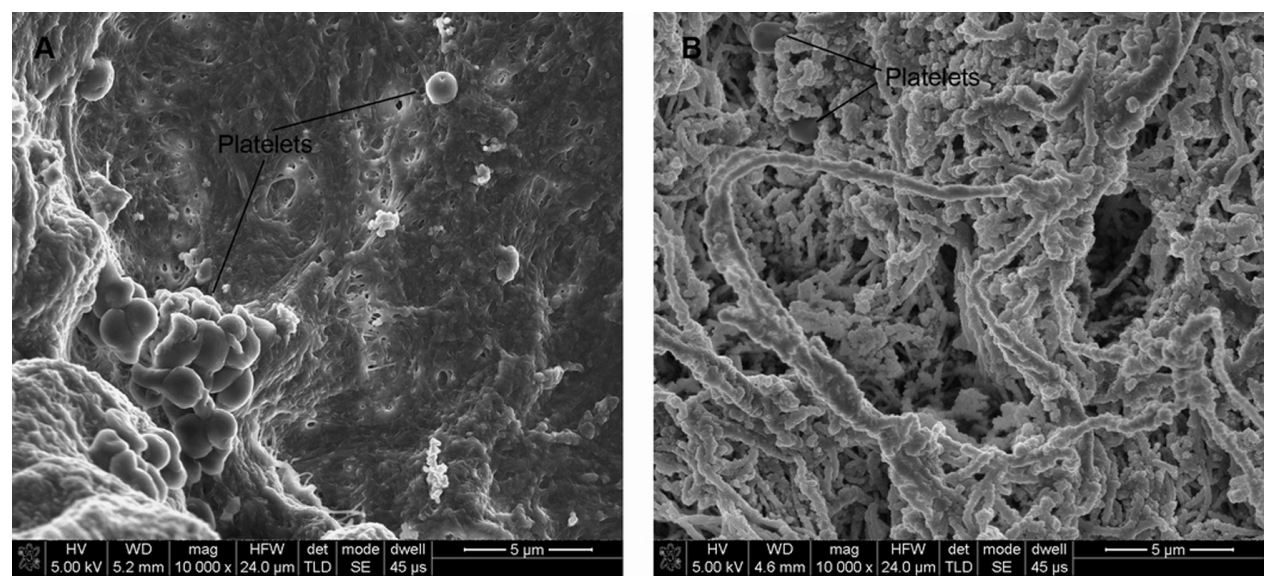

Figure 7 SEM images (5000x) of platelets adhering to the surface of unmodified (A) and heparin/DHI-modified bovine jugular vein scaffolds (B). Note: The arrows indicate adhering platelets.

Abbreviations: DHI, dihydroxy-iron; SEM, scanning electron microscope.

biomechanical stability of the scaffold, which might be because the heparin/DHI complexes form crossbridges and crosslink adjacent fibrils. However, there was no significant difference in biomechanical stability between scaffolds modified by ten and 13 cycles of heparin/DHI, indicating that the crosslinked heparin/DHI complexes have limited biomechanical stability. Increasing the number of assembly cycles beyond ten may not increase the biomechanical stability of the scaffold further. The modified scaffold was stiffer than its unmodified counterpart, but softer than the glutaraldehyde-treated scaffold.

The modified scaffolds used in this study released heparin in a sustained manner during 8 weeks of incubation in phosphate-buffered saline. The amount of heparin released from a $1 \mathrm{~cm}^{2}$ area of scaffold sheets modified by four, seven, and ten cycles of heparin/DHI after 8 weeks of washing was approximately $1.88 \mathrm{mg}(58 \%), 1.91 \mathrm{mg}(35 \%)$, and $2.01 \mathrm{mg}(23 \%)$, respectively. These results indicate that the heparin/DHI complexes were firmly linked to the BJV scaffolds, and more linked heparin enabled heparin release for a longer period of time. The prothrombin time and APTT of the modified BJV sheets after washing for one day and 8 weeks were above the upper limit of the normal reference ranges, indicating that heparin/DHI-modified BJV scaffolds retained antithrombogenic activity for at least 8 weeks. Therefore, modification with heparin/DHI complexes could provide a stable antithrombogenic surface for acellular BJV scaffolds and effectively resist thrombus formation. All these observations indicate that heparin/DHI-modified
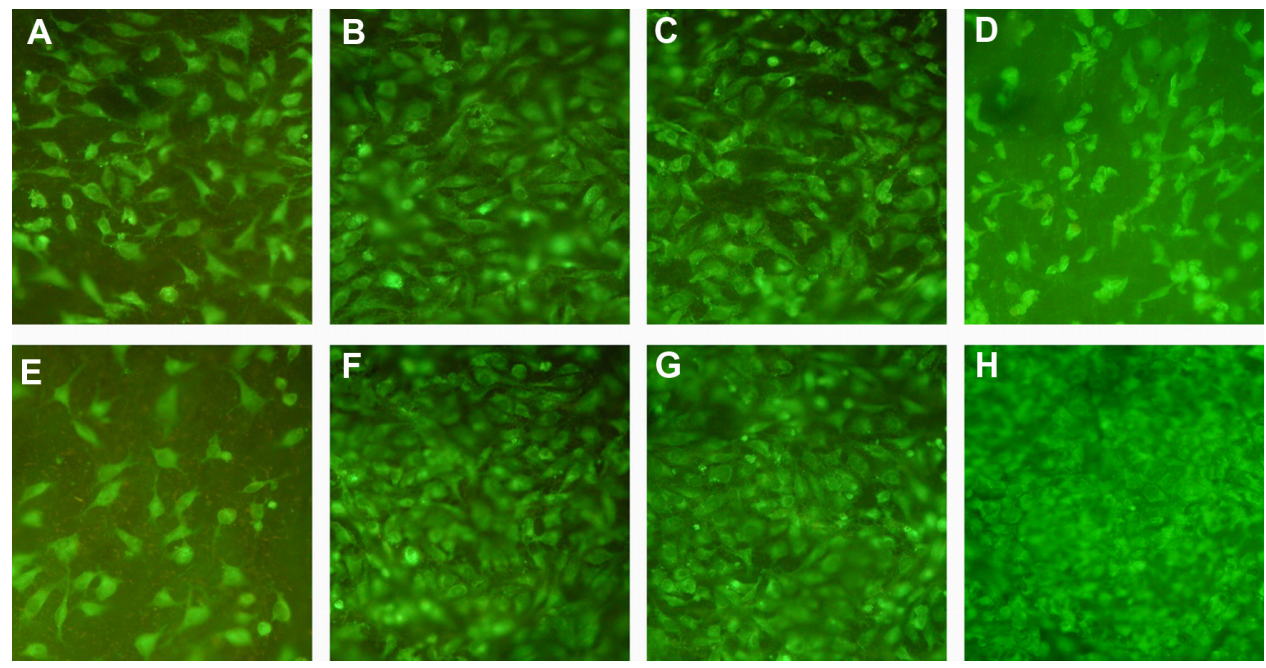

Figure $8 \mathrm{DioC}_{18}$ staining of endothelial cells on the luminal surface of scaffolds (200x). (A-D) are photographs of endothelial cells on the luminal surface of unmodified scaffolds after incubation for one, 4, 7 and 10 days, respectively. (E-H) are photographs of endothelial cells on the luminal surface of heparin/DHI-modified scaffolds after incubation for one, 4, 7 and 10 days, respectively. Abbreviation: $\mathrm{DHI}$, dihydroxy-iron. 

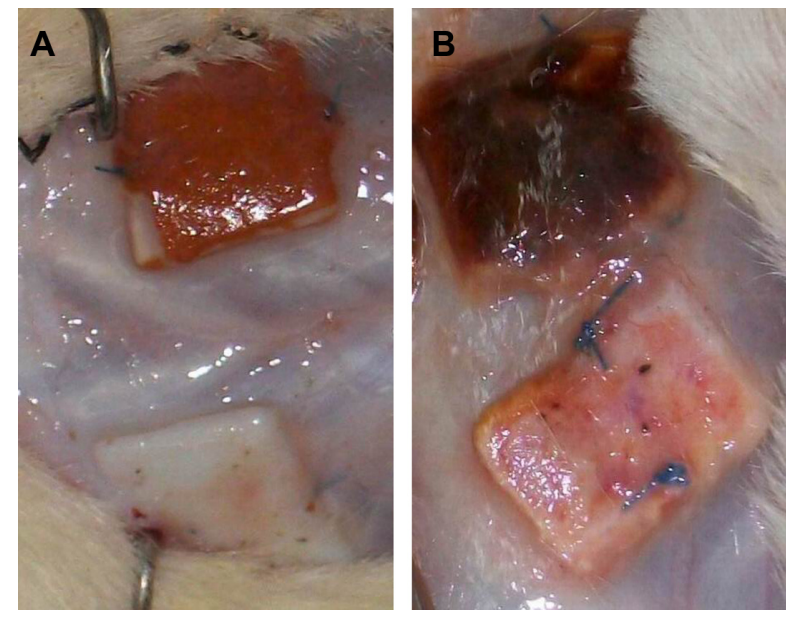

Figure 9 Gross appearance of unmodified and heparin/DHI-modified scaffold sheets at 30 days (A) and 60 days (B) after implantation.

Note: The modified scaffolds retain their golden yellow color.

Abbreviation: DHI, dihydroxy-iron.

scaffolds might be more antithrombogenic than unmodified scaffolds.

Platelet adhesion increases the likelihood of mural thrombosis, so less platelet adhesion means less thrombogenicity. Compared with the unmodified scaffolds, adhesion of platelets to the surface of the heparin/DHI-modified scaffolds was dramatically decreased in this study, suggesting that the modified scaffolds should have better antithrombogenicity than the unmodified scaffolds.

It has been reported that iron ions and heparin molecules can protect biomaterials from calcification. ${ }^{36-38}$ We obtained similar results in our experiment. The calcium content of the heparin/DHI-modified scaffolds decreased significantly compared with that in the unmodified scaffolds, suggesting that the modified BJV scaffolds may reduce calcification.

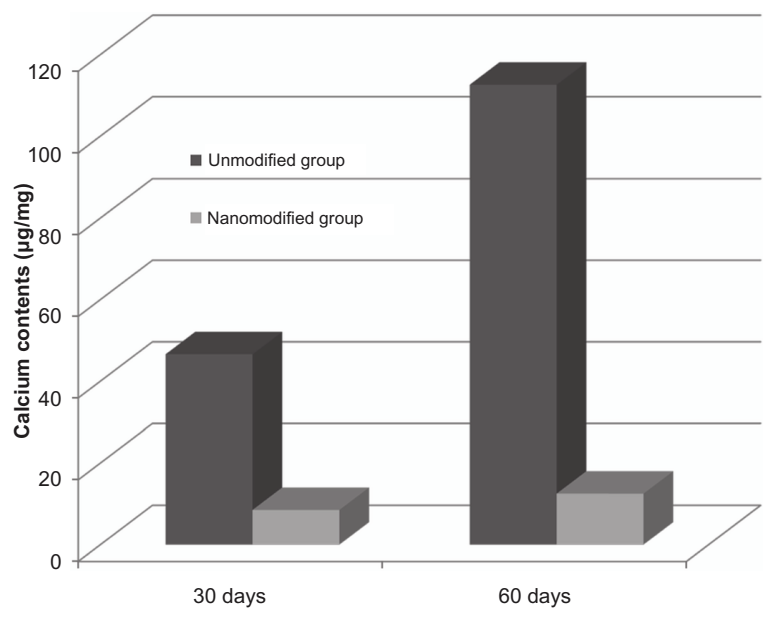

Figure 10 Column graphs showing the calcium content of unmodified and nanomodified scaffolds.
In the current study, the number of endothelial cells on the surface of the heparin/DHI-modified scaffold sheets was less than the number on the surface of the unmodified sheets in the early period of culture, but endothelial cells could grow and proliferate on the luminal surface of the modified scaffolds more rapidly, and the number of endothelial cells on the surface of heparin/DHI-modified scaffolds was greater than that on the surface of the unmodified scaffolds after 10 days of incubation. Furthermore, growth of endothelial cells on the unmodified scaffolds had slowed down and stopped by 10 days of incubation. These findings indicate that nanomodification using heparin/DHI decreased adherence of endothelial cells in the initial period, but once adhered, these cells grew and proliferated rapidly on the luminal surface in vitro. Further, the heparin/DHI complexes were not cytotoxic.

Heparin molecules and DHI ions can be alternately linked to acellular vascular scaffolds and have four main actions. First, heparin can reduce the thrombogenicity of vascular xenografts and prevent formation of blood clots on the surface after implantation. ${ }^{14}$ Second, coating the graft with heparin provides a substrate to bind growth factors, such as basic fibroblast growth factor, epithelial growth factor, and vascular endothelial growth factor, that may accelerate endothelialization and regeneration. ${ }^{18,39}$ Third, DHI ions and heparin molecules can reduce calcification of biomaterials, and this may minimize calcification in vascular xenografts. Fourth, heparin/DHI complexes can crosslink adjacent fibrils and strengthen the biomechanical stability of acellular tissueengineered scaffolds.

The amount of heparin linked to the scaffolds increases with an increase in the number of assembly cycles. However, the optimal number of assembly cycles and amount of heparin that needs to be linked for maximum efficacy are not clear. Although heparin inhibits smooth muscle cell proliferation and neointimal formation, resists inflammation, and has other beneficial effects, it is not clear whether heparin/ DHI-modified acellular vascular scaffolds have these same properties. ${ }^{16,40,41}$ These issues still need further evaluation, and additional studies with heparin/DHI-nanomodified tissue-engineered vascular grafts implanted in animals are in progress.

\section{Conclusion}

We have created heparin-nanomodified acellular vascular xenografts by alternating dative covalent linkage of heparin and DHI on acellular BJV scaffolds via layer-by-layer selfassembly. Our initial results show that acellular vascular scaffolds modified with heparin/DHI complexes have excellent 
antithrombogenicity and biomechanical stability, resist calcification, decrease platelet adhesion, and stimulate proliferation of endothelial cells without cytotoxicity. This study suggests a new method for developing tissue-engineered vascular grafts. However, these results are preliminary, and further in vivo studies in animals with long-term implantation of these heparin/DHI-modified grafts are needed.

\section{Acknowledgments}

This project was supported financially by the National 863 Plan for the People's Republic of China (2007AA071900), the National Natural Science Foundation of China (81071275), and the Natural Science Foundation of the Jiang'xi Province of China (20122BAB205098).

\section{Disclosure}

The authors report no conflicts of interest in this work.

\section{References}

1. Torikai K, Ichikawa H, Hirakawa K, et al. A self-renewing, tissueengineered vascular graft for arterial reconstruction. Thorac Cardiovasc Surg. 2008;136:37-45.

2. Teebken OE, Haverich A. Tissue engineering of small diameter vascular grafts. Eur J Vasc Endovasc Surg. 2002;23:475-485.

3. Cooper GJ, Underwood MJ, Deverall PB. Arterial and venous conduits for coronary artery bypass: a current review. Eur J Cardiothorac Surg. 1996;10:129-140.

4. Luong-van E, GrondahI L, Chua KN, Leong KW, Nurcombe V, Cool SM Controlled release of heparin from poly (epsilon-caprolactone) electrospun fibers. Biomaterials. 2006;27:2042-2050.

5. Lu WD, Yu FL, Wu ZS. Superior vena cava reconstruction using bovine jugular vein conduit. Eur J Cardiothorac Surg. 2007;32:816-817.

6. Lu WD, Zhang M, Wu ZS, Hu TH. Decellularized and photooxidatively crosslinked bovine jugular veins as potential tissue engineering scaffolds. Interact Cardiovasc Thorac Surg. 2009;8:301-315.

7. Lu WD, Zhang M, Wu ZS, et al. The performance of photooxidatively crosslinked acellular bovine jugular vein conduits in the reconstruction of connections between pulmonary arteries and right ventricles. Biomaterials. 2010;31:2934-2943.

8. Schmidt CE, Baier JM. Acellular vascular tissues: natural biomaterials for tissue repair and tissue engineering. Biomaterials. 2000;21: 2215-2231.

9. Tschoeke B, Flanagan TC, Cornelissen A, et al. Development of a composite degradable/nondegradable tissue-engineered vascular graft. Artif Organs. 2008;32:800-809.

10. Rastan AJ, Walther T, Daehnert I, et al. Bovine jugular vein conduit for right ventricular outflow tract reconstruction: evaluation of risk factors for mid-term outcome. Ann Thorac Surg. 2006;82:1308-1315.

11. Ozkan S, Akay TH, Gultekin B, Sezgin A, Tokel K, Aslamaci S. Xenograft transplantation in congenital cardiac surgery at Baskent Iniversity: midterm results. Transplant Proc. 2007;39:1250-1254.

12. Fiore AC, Ruzmetov M, Huynh D, et al. Comparison of bovine jugular vein with pulmonary homograft conduits in children less than 2 years of age. Eur J Cardiothoracic Surg. 2010;38:318-325.

13. Crikis S, Cowan PJ, d'Apice AJ. Intravascular thrombosis in discordant xenotransplantation. Transplantation. 2006;82:1119-1123.

14. Liao D, Wang X, Lin PH, Yao Q, Chen CJ. Covalent linkage of heparin provides a stable anti-coagulation surface of decellularized porcine arteries. J Cell Mol Med. 2009;13:2736-2743.
15. Rabenstein DL. Heparin and heparan sulfate: structure and function. Nat Prod Rep. 2002;19:312-331.

16. Wang XN, Chen CZ, Yang M, Gu YJ. Implantation of decellularized small-caliber vascular xenografts with and without surface heparin treatment. Artif Organs. 2007;31:99-104.

17. Basmadjian D, Sefton MV. Relationship between release rate and surface concentration for heparinized materials. J Biomed Mater Res. 1983; 17:509-518

18. Tan Q, Tang H, Hu J, et al. Controlled release of chitosan/heparin nanoparticle-delivered VEGF enhances regeneration of decellularized tissue-engineered scaffolds. Int J Nanomedicine. 2011;6:929-942.

19. Lee WK, Park KD, Han DK, Suh H, Park JC, Kim YH. Heparinized bovine pericardium as a novel cardiovascular bioprosthesis. Biomaterials. 2000;21:2323-2230.

20. Wissink MJ, Beernink R, Pieper JS, et al. Immobilization of heparin to EDC/NHS-crosslinked collagen. Characterization and in vitro evaluation. Biomaterials. 2001;22:151-163.

21. Yu SH, Wu YB, Mi FL, Shyu SS. Polysaccharide-based artificial extracellular matrix: preparation and characterization of threedimensional, macroporous chitosan, and heparin composite scaffold. J Appl Polym Sci. 2008;109:3639-3644.

22. Liu M, Yue X, Dai Z, et al. Novel thrombo-resistant coating based on iron-polysaccharide complex multilayers. ACS Appl Mater Interfaces. 2009;1:113-123.

23. Balamurugan $\mathrm{K}$, Kalaichelvan $\mathrm{AD}$. Isolation and identification of heparin like substances from the body tissue of conus musicus. Int $J$ Pharm Tech Res. 2009;1:1650-1653.

24. Ahola MS, Säilynoja ES, Raitavuo MH, Vaahtio MM, Salonen JI, YliUrpo AU. In vitro release of heparin from silica xerogels. Biomaterials. 2001;22:2163-2170.

25. Smith PK, Mallia AK, Hermanson GT. Colorimetric method for the assay of heparin content in immobilized heparin preparations. Anal Biochem. 1980;109:466-473.

26. Okrój W, Walkowiak-Przybyło M, Rośniak-Bak K, Klimek L, Walkowiak B. Comparison of microscopic methods for evaluating platelet adhesion to biomaterial surfaces. Acta Bioeng Biomech. 2009;11: $45-49$.

27. Emeis JJ, Edgell CJ. Fibrinolytic properties of a human endothelial hybrid cell line (Ea.hy 926). Blood. 1988;71:1669-1675.

28. Zhao X, Kim J, Cezar CA, et al. Active scaffolds for on-demand drug and cell delivery. Proc Natl Acad Sci U S A. 2011;108:67-72.

29. Pettenazzo E, Valente M, Thiene G. Octanediol treatment of glutaraldehyde fixed bovine pericardium: evidence of anticalcification efficacy in the subcutaneous rat model. Eur J Cardiothorac Surg. 2008;34: 418-422.

30. Nugent MA. Heparin sequencing brings structure to the function of complex oligosaccharides. Proc Natl Acad Sci U S A. 2000;97: 10301-10303.

31. Sasisekharan S, Venkataraman G. Heparin and heparan sulfate: biosynthesis, structure and function. Curr Opin Chem Biol. 2000;4: 626-631.

32. Wagenseil JE, Mecham RP. Vascular extracellular matrix and arterial mechanics. Physiol Rev. 2009;89:957-989.

33. Tao Y, Hu T, Wu Z, Tang H, Hu Y, Tan Q. Heparin-iron complex multilayer nanomodification improves hemocompatibility of decellular xenograft. Zhong Nan Da Xue Xue Bao Yi Xue Ban. 2012;37:260-266. Chinese.

34. Von der Mark K, Park J, Bauer S, Schmuki P. Nanoscale engineering of biomimetic surfaces: cues from the extracellular matrix. Cell Tissue Res. 2010;339:131-153.

35. Shekaran A, Garcia AJ. Nanoscale engineering of extracellular matrixmimetic bioadhesive surfaces and implants for tissue engineering Biochim Biophys Acta. 2011;1810:350-360.

36. Baldwin MT, Ciesliga BL, Barkasi LD, Webb CL. Long-term anticalcification effect of $\mathrm{Fe} 3+$ in rat subdermal implants of glutaraldehyde preserved bovine pericardium. ASAIO Trans. 1991;37: $170-172$. 
37. Valdes TI, Moussy F. A ferric chloride pre-treatment to prevent calcification of Nafion membrane used for implantable biosensors. Biosens Bioelectron. 1999;14:579-585.

38. Vasudev SC, Moses LR, Sharma CP. Covalently bonded heparin to alter the pericardial calcification. Artif Cells Blood Substit Immobil Biotechnol. 2000;28:241-253.

39. Singh S, Wu BM, Dunn JC. The enhancement of VEGF-mediated angiogenesis by polycaprolactone scaffolds with surface cross-linked heparin. Biomaterials. 2011;32:2059-2069.
40. Garg HG, Mrabat H, Yu L, et al. Effect of carboxyl-reduced heparin on the growth inhibition of bovine pulmonary artery smooth muscle cells. Carbohydr Res. 2010;345:1084-1087.

41. Beamish JA, Geyer LC, Haq-Siddiqi NA, Kottke-Marchant K, Marchant RE. The effects of heparin releasing hydrogels on vascular smooth muscle cell phenotype. Biomaterials. 2009;30:6286-6294.

\section{Publish your work in this journal}

The International Journal of Nanomedicine is an international, peerreviewed journal focusing on the application of nanotechnology in diagnostics, therapeutics, and drug delivery systems throughout the biomedical field. This journal is indexed on PubMed Central, MedLine, CAS, SciSearch $\AA$, Current Contents ${ }^{\circledR} /$ Clinical Medicine,
Journal Citation Reports/Science Edition, EMBase, Scopus and the Elsevier Bibliographic databases. The manuscript management system is completely online and includes a very quick and fair peer-review system, which is all easy to use. Visit http://www.dovepress.com/ testimonials.php to read real quotes from published authors. 